\title{
Patrón de caries dental en niños de 3-5 años de un Hogar infantil. Medellín, Colombia
}

Pattern of dental caries in 3-5 year old children in a children's day care center. Medellín, Colombia

Padrão de cárie dentária em crianças de 3 a 5 anos em um Lar infantil. Medellín, Colombia

\section{Gloria Escobar-Paucar ${ }^{1}$ Blanca Susana Ramirez-Puerta²}

\begin{abstract}
Recibido: 9 de junio de 2020
Aprobado: 31 de agosto de 2020

Publicado: 15 de noviembre de 2020

Cómo citar este artículo:

Escobar-Paucar G, Ramírez-Puerta BS. Patrón de caries dental en niños de 3-5 años de un Hogar infantil. Medellín, Colombia. Revista Nacional de Odontología. (2020); 16(2), 1-14. doi: https://doi.org/10.16925/2357-4607.2020.02.03
\end{abstract}

Artículo de investigación. https://doi.org/10.16925/2357-4607.2020.02.03

1 Master in Public Health. School of Dentistry, Universidad de Antioquia. Facultad de Odontología, Universidad de Antioquia Calle 64 N 52-59, Medellín, Antioquia.

Correo electrónico: matilde.escobar@udea.edu.co.

ORCID: https://orcid.org/0000-0002-4721-4749

2 Master in Epidemiology. School of Dentistry, Universidad de Antioquia.

ORCID: https://orcid.org/0000-0002-0322-1058 


\section{Resumen}

Introducción y objetivo: la caries dental en población preescolar requiere mayores esfuerzos para reconocer el problema y diseñar estrategias de intervención. El objetivo fue identificar experiencia y patrón de distribución de las lesiones de caries dental en niños asistentes a un Hogar Infantil de la ciudad.

Materiales y métodos: una odontóloga calibrada examinó 177 niños de 3 a 5 años, matriculados en un centro infantil en 2018. Se utilizaron criterios ICDAS. Se calcularon la experiencia de caries de la infancia temprana (CIT) y caries severa de la infancia temprana (CIT-S). Los indicadores $\mathrm{C}_{5-6 \text { ICDAS }}$ opd, $\mathrm{C}_{5-6 \text { ICDAS }}$ ops, $\mathrm{C}_{1-6 \text { ICDAS }}$ opd, $\mathrm{C}_{1-6 \text { ICDAS }}$ ops; así como los porcentajes de dientes y superficies individuales afectadas y por pares de dientes homólogos.

Resultados: el 61 \% tenía CIT y el 48 \% presentaba CIT-S, con IC $95 \%(53,5 ; 68,5$ y 40,4; 55,7) respectivamente. El indicador $\mathrm{C}_{1-6 \text { ICDAs }}$ opd fue $3,3 \pm 4,9$ a los tres años y $5,3 \pm 4,8$ a los cinco años. Los molares primarios mostraron mayor experiencia de caries dental (32,8 \% a 45,2 \%), especialmente en oclusal (25,3\% a 33,3\%). Los dientes menos afectados fueron los anteriores inferiores (1,7 \% a 2,8 \%). La experiencia de caries en dientes homólogos mostró diferencias estadísticamente significativas entre las superficies oclusales y las demás superficies en molares. En incisivos y caninos, la superficie bucal mostró mayor experiencia de caries.

Conclusión: la caries dental afecta la mayoría de niños y niñas de una institución en Medellín, con una distribución de lesiones similar a otros estudios. Es necesario seguir trabajando para implementar estrategias de detección y control oportuno de la enfermedad, de acuerdo con el patrón encontrado.

Palabras clave: caries dental, dentición primaria, salud bucal.

\section{Abstract}

Introduction: Dental caries in preschool children, a pending health challenge, requires greater efforts to recognize the problem and to design intervention strategies. The purpose of this study was to identify experience and pattern distribution of dental caries lesions in children who attend a kindergarten in the city.

Materials and methods: A calibrated dentist examined 177 children 3-to 5-year-old, using ICDAS criteria. Caries experience (ECC) and severe early childhood caries (ECC-S) was calculated; the indexes $\mathrm{d}_{5-6}$ ICDASmft, $d_{5-6}$ ICDASmfs, $d_{1-6}$ ICDASmft, $d_{1-6}$ ICDASmfs; as well as the percentages of affected teeth and individual surfaces and by homologous teeth.

Results: A $61 \%$ had ECC and 48\% ECC-S with 95\% Cl $\left(53.5 ; 68.5\right.$ and 40.4; 55.7) respectively. The $\mathrm{d}_{1-6}$ ICDASmft index was $3.3 \pm 4.9$ in 3 -year-old children and $5.3 \pm 4.8$ in 5-year-old. Primary molars showed greater dental caries experience (32.8\% to $45.2 \%)$, especially in occlusal $(25.3 \%$ to $33.3 \%)$. The least affected teeth were the lower anterior (1.7\% to $2.8 \%)$. The caries experience in homologous teeth showed statistically significant differences between the occlusal surfaces and the other surfaces in molars. In the incisors and canines, the buccal surface showed greater caries experience.

Conclusion: Dental caries affects the majority of children who attend an institution in Medellín, with a distribution of lesions similar to other studies; It is necessary to continue working to implement strategies for detection and timely control of the disease, according to the pattern found.

Keywords: Dental Caries, Primary Dentition, Oral Health.

\section{Resumo}

Introdução: a cárie dentária em crianças pré-escolares, um desafio de saúde pendente, requer maiores esforços para reconhecer o problema e desenhar estratégias de intervenção. O objetivo deste estudo foi identificar a experiência e Distribuição do padrão de lesões de cárie dentária em crianças que frequentam uma creche da cidade. 
Materiais e métodos: Um dentista calibrado examinou 177 crianças de 3 a 5 anos de idade, usando os critérios do ICDAS. A experiência de cárie (ECC) e cárie severa na primeira infância (ECC-S) foi calculada; os índices d5-6ICDASmft, d5-6ICDASmfs, d1-6ICDASmft, d1-6ICDASmfs; bem como as porcentagens de dentes afetados e superfícies individuais e por dentes homólogos.

Resultados: A 61\% apresentou CEC e 48\% CEC-S com IC 95\% (53,5; 68,5 e 40,4; 55,7), respectivamente. 0 d1-6 ICDASmft o índice foi de 3,3 \pm 4,9 em crianças de 3 anos e 5,3 $\pm 4,8$ em crianças de 5 anos. Os molares decíduos apresentaram maior dentição experiência de cárie (32,8\% a 45,2\%), principalmente na oclusal (25,3\% a 33,3\%). Os dentes menos afetados foram os anterior inferior (1,7\% a 2,8\%). A experiência de cárie em dentes homólogos mostrou diferenças estatisticamente significativas entre as superfícies oclusais e as outras superfícies nos molares. Nos incisivos e caninos, o vestibular superfície mostrou maior experiência de cárie.

Conclusão: A cárie dentária afeta a maioria das crianças que frequentam uma instituição em Medellín, com uma distribuição de lesões semelhantes a outros estudos; É preciso continuar trabalhando para implementar estratégias de detecção e controle oportuno da doença, de acordo com o padrão encontrado.

Palavras-chave: Cárie Dentária, Dentição Primária, Saúde Bucal.

\section{Introducción}

Los esfuerzos por mejorar las condiciones de salud bucal de niños y niñas menores de seis años, cuyo mayor desafío es la presencia de caries dental desde edades muy tempranas, requieren la articulación de acciones y esfuerzos intersectoriales orientados al control de la enfermedad (1). Para un grupo de expertos, la Caries de la Infancia Temprana (CIT) es una enfermedad prevenible y constituye una carga innecesaria para la sociedad, por lo cual sugieren un manejo apropiado por parte de los padres, los profesionales de la salud y los agentes comunitarios, así como políticas basadas en la evidencia para reducir el reto que ésta representa $(2,3)$.

La Academia Americana de Odontología Pediátrica (AAPD) promueve la educación tanto de la comunidad como del personal de salud y recomienda que cuando se identifica la presencia de caries dental se realice la remisión inmediata para implementar medidas oportunas que, en lo posible, reduzcan la necesidad de tratamientos de tipo operatorio (4). Lo anterior cobra mayor vigencia en tanto se puede interferir con el proceso dinámico de la caries dental en todas las etapas de la lesión, concepto conocido como Control de Caries $(5,6)$.

De otro lado, aunque las lesiones se pueden desarrollar en cualquier superficie y algunos autores afirman que no existen partes de un diente más resistentes o susceptibles a la enfermedad debido a su composición química o estructural, se sabe que las lesiones se desarrollan en sitios donde se permite que la biopelícula se acumule y madure (7). Ello explica que no todos los dientes son afectados de igual manera, y es posible identificar ciertos patrones de distribución de las lesiones en dentición primaria (8-13). 
Conocer esta información puede ser de gran utilidad para llamar la atención a los cuidadores, los agentes comunitarios, el personal de equipos primarios de salud y los odontólogos, sobre aquellos dientes o superficies donde puede desarrollarse la caries dental, e identificar la presencia de lesiones. De esta manera se puede contribuir a la integración de acciones oportunas para la prevención y control de la enfermedad, un aspecto en el cual enfatiza el llamado reciente de la Organización Mundial de la Salud para hacer frente a este reto que afecta la salud infantil (3).

El objetivo del estudio fue identificar la experiencia y el patrón de distribución de las lesiones de caries dental en niños matriculados en un hogar infantil de la ciudad de Medellín.

\section{Materiales y métodos}

Estudio descriptivo transversal realizado en 177 niños de un total de 187 con edades de 3 a 5 años, matriculados en un hogar infantil de la ciudad de Medellín en 2018. Un niño no aceptó ser examinado y nueve no asistieron a la valoración clínica, el criterio de exclusión fue la falta de consentimiento de los padres o la no aceptación del niño. El estudio contó con la autorización de las directivas y el consentimiento informado de cada una de las familias de los niños participantes. Se obtuvo aprobación mediante el Concepto 13-2017 en el Acta 2 de 2017 por el Comité de Bioética de la institución.

El registro del estado dental se hizo con base en el Sistema Internacional de Detección y Valoración de Caries - ICDAS (14), que comprende seis niveles de avance de las lesiones. Para el registro del código de caries se siguió la recomendación de unir los niveles 1 y 2 de lesiones limitadas al esmalte dental - ICDAS EPI (15). El examen clínico fue realizado por una odontóloga calibrada en el Sistema ICDAS (Kappa intraexaminador 0,77 e interexaminador 0,71). La valoración de cada una de las superficies dentales se hizo mediante inspección visual y se usó la sonda 11.5B (WHO) para confirmar el diagnóstico cuando se consideró necesario. El examen se realizó en el hogar infantil utilizando silla odontológica, lámpara y compresor portátil. Previo al examen, se realizó cepillado dental. Se aisló el área de examen mediante el uso de rollos de algodón y las superficies dentales se secaron con gasa y aire. Se utilizó un formato que permite hacer un registro de los criterios ICDAS para cada superficie presente, y a partir de este calcular el indicador tradicional copd, como lo reportó el IV Estudio Nacional de Salud Bucal.

Los datos fueron analizados con el programa estadístico IBM-SPSS ${ }^{\circledR}$ versión 23.0 y en el programa Excel. Se calcularon los índices $\mathrm{C}_{5-6 I C D A S}$ Opd, $\mathrm{C}_{5-6 I C D A S}$ Ops, $\mathrm{C}_{1-6 \text { ICDAS }}$ Opd, $\mathrm{C}_{1-\text {-IICDAS }}$ ops y los intervalos de confianza del 95\%. Para el cálculo de los 
porcentajes de niños con caries de la infancia temprana (CIT) y caries severa de la infancia temprana (CIT-S), se tuvieron en cuenta las guías de la AAPD. Estas definen CIT como la presencia de una o más superficies de dientes primarios con caries cavitada o no, obturada o perdida por caries, en niños menores de 6 años, y establece como CIT-S cuando hay algún signo de caries en superficies lisas en niños menores de 3 años, y cuando en los de 3 a 5 años hay una o más superficies lisas de los dientes primarios anteriores superiores con lesiones cavitadas, obturadas o perdidas por caries, o si el número de superficies cariadas (incluyendo lesiones no cavitadas), obturadas o perdidas por caries es $\geq 4$ a los tres años, $\geq 5$ a los 4 años y $\geq 6$ a los 5 años de edad (4).

Se calculó el porcentaje de dientes y superficies dentales con experiencia de caries dental para todos los niños examinados y para cada una de las edades. Adicionalmente, se estimó el porcentaje de experiencia para dientes homólogos, y con el programa estadístico Epidat 3.1 se analizaron diferencias entre superficies dentales de cada par de dientes, mediante la prueba $\mathrm{Chi}^{2}$ o prueba exacta de Fisher.

\section{Resultados}

Se evaluaron 177 niños y niñas con edades entre los tres y los cinco años, con un

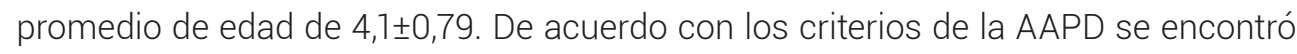
que el $61 \%$ tenía caries de la infancia temprana (CIT), valor que aumenta de $46 \%$ en el grupo de 3 años de edad, a 70 \% en el grupo de cinco años. En cuanto a caries severa de la infancia temprana (CIT-S), se encontró en el $48 \%$ de todos los niños evaluados, con valores de 36,0 \%, 49,3 \% y 56,7 \% en los niños de 3, 4 y 5 años, respectivamente.

Para comparación con otros estudios, se calculó el indicador de experiencia de caries dental de acuerdo con los criterios del copd tradicional (16) que solo considera dientes que tienen lesiones con cavidad evidente en dentina, obturados o perdidos por caries. Se observó que el 27,1 \% de los niños examinados han tenido experiencia de caries dental, y a los cinco años de edad, este valor fue de $36,7 \%$.

A los tres años de edad, los niños tienen en promedio $3,3 \pm 4,9$ dientes y $5,2 \pm 8,5$ superficies afectados por caries dental, índices $\mathrm{C}_{\text {1-6 ICDAS }}$ opd y $\mathrm{C}_{1-6 \text { ICDAS }}$ ops; valores que alcanzan respectivamente $5,3 \pm 4,8$ y 10,0 $\pm 14,3$ a los cinco años, (tabla 1). 
6 Patrón de caries dental en niños de 3-5 años de un Hogar infantil. Medellín, Colombia

Tabla 1. Distribución de niños con caries de la infancia temprana (CIT) y con caries severa de la infancia temprana (CIT-S) y promedios de índices $\mathrm{C}_{1-6 \text { ICDAS }}$ opd y $\mathrm{c}_{1-6 \text { ICDAS }}$ ops

\begin{tabular}{|c|c|c|c|c|c|c|c|c|c|c|c|c|c|}
\hline \multirow{2}{*}{$\begin{array}{c}\text { Edad } \\
\text { (años) }\end{array}$} & \multicolumn{3}{|c|}{ Copd $>0$ tradicional $\S$} & \multicolumn{3}{|c|}{$\mathrm{CIT}^{*}$} & \multicolumn{3}{|c|}{ CIT-S S $^{\star \star}$} & \multicolumn{2}{|c|}{$c_{1-61 \text { ICAS }}$ opd $\dagger$} & \multicolumn{2}{|c|}{$c_{1-6 \text { ICDAS }}$ ops †† } \\
\hline & $n^{\circ}$ & $\%$ & IC & $\mathbf{n}^{\circ}$ & $\%$ & IC & $n^{\circ}$ & $\%$ & IC & $\begin{array}{c}\text { Media } \\
\text { (DE) }\end{array}$ & IC & $\begin{array}{c}\text { Media } \\
\text { (DE) }\end{array}$ & IC \\
\hline \multirow{2}{*}{$\begin{array}{l}\text { Tres } \\
(n=50)\end{array}$} & \multirow{2}{*}{6} & \multirow{2}{*}{12} & \multirow{2}{*}{3,$0 ; 21,0$} & \multirow{2}{*}{23} & \multirow{2}{*}{46,0} & \multirow{2}{*}{31,$2 ; 60,8$} & \multirow{2}{*}{18} & \multirow{2}{*}{36,0} & \multirow{2}{*}{21,$7 ; 50,3$} & 3,3 & \multirow{2}{*}{1,$9 ; 4,7$} & 5,2 & \multirow{2}{*}{2,$7 ; 7,6$} \\
\hline & & & & & & & & & & $(4,9)$ & & $(8,5)$ & \\
\hline \multirow{2}{*}{$\begin{array}{l}\text { Cuatro } \\
(\mathrm{n}=67)\end{array}$} & \multirow{2}{*}{20} & \multirow{2}{*}{29,9} & \multirow{2}{*}{18,$9 ; 40,9$} & \multirow{2}{*}{43} & \multirow{2}{*}{64,2} & \multirow{2}{*}{51,$9 ; 76,4$} & \multirow{2}{*}{33} & \multirow{2}{*}{49,3} & \multirow{2}{*}{36,$5 ; 61,9$} & 5,0 & \multirow{2}{*}{3,$8 ; 6,3$} & 8,0 & \multirow{2}{*}{5,$6 ; 10,4$} \\
\hline & & & & & & & & & & $(5,2)$ & & $(9,7)$ & \\
\hline \multirow{2}{*}{$\begin{array}{l}\text { Cinco } \\
(n=60)\end{array}$} & \multirow{2}{*}{22} & \multirow{2}{*}{36,7} & \multirow{2}{*}{24,$5 ; 48,9$} & \multirow{2}{*}{42} & \multirow{2}{*}{70,0} & \multirow{2}{*}{57,$6 ; 82,4$} & 34 & 567 & 43.3 .70 & 5,3 & 40.65 & 10,0 & 63.136 \\
\hline & & & & & & & 34 & 56,1 & $43,3,10,0$ & $(4,8)$ & $4,0,6,5$ & 14,3 & 0,3,13,6 \\
\hline Total & 48 & 27.1 & $20.6 \cdot 336$ & 108 & 610 & 53.5 .68 .5 & 85 & 48,0 & $40,4: 55,7$ & 4,6 & $3,9: 5,4$ & 7,9 & 6,$2 ; 9,5$ \\
\hline $\mathrm{n}=177)$ & 40 & 21,1 & $20,0,03,0$ & 100 & 01,0 & נ, & & & & $(5,0)$ & (3,j, & 11,3 & \\
\hline
\end{tabular}

${ }^{*}$ : Caries de la infancia temprana (AAPD)

${ }^{* *}$ : Caries severa de la infancia temprana (AAPD)

t: Índice de dientes cariados (incluyendo todas las lesiones de caries), obturados y perdidos por caries

†t: Índice de superficies cariadas (incluyendo todas las lesiones de caries), obturadas y perdidas por caries

IC: Intervalo de confianza 95\%

(DE): desviación estándar

§: \% de niños con experiencia de caries dental cavitada en dentina

Fuente: elaboración propia

Las superficies oclusales presentaron los porcentajes más altos de experiencia de caries dental, con valores entre $25,3 \%$ y 33,3 \% en los diferentes grupos etarios, y las superficies dentales menos afectadas fueron las de dientes anteriores inferiores (figura 1). 
a) Total ( $n=177)$

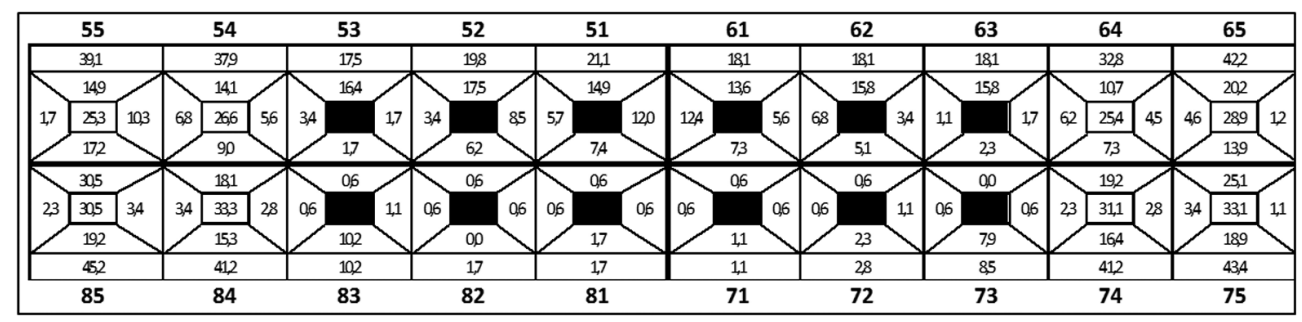

b) Tres años ( $n=50)$

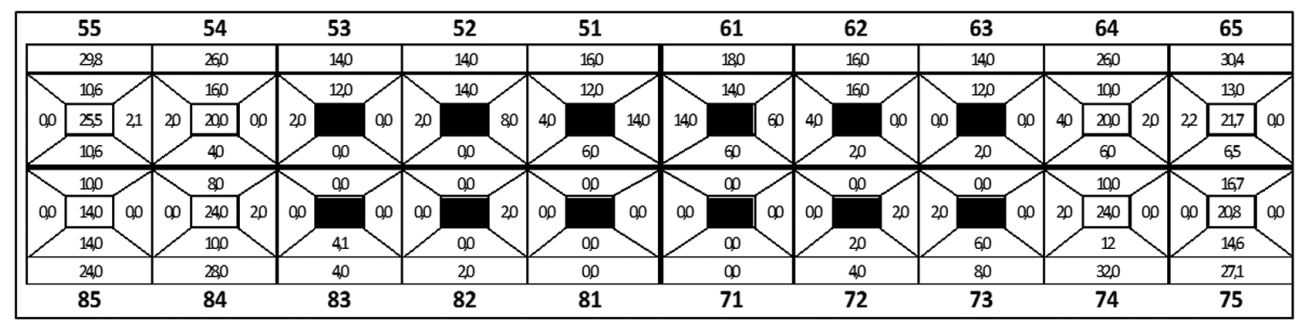

c) Cuatro años ( $n=67)$

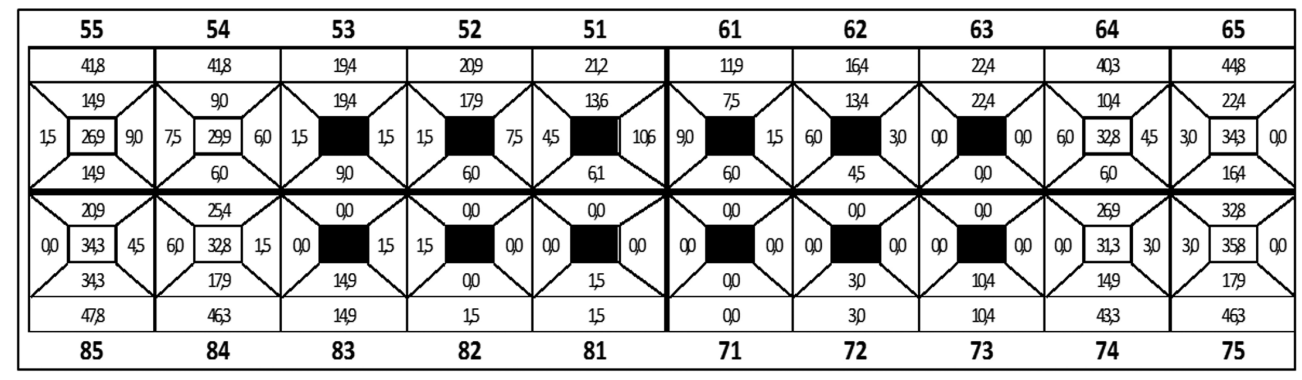

d) Cinco años ( $n=60)$

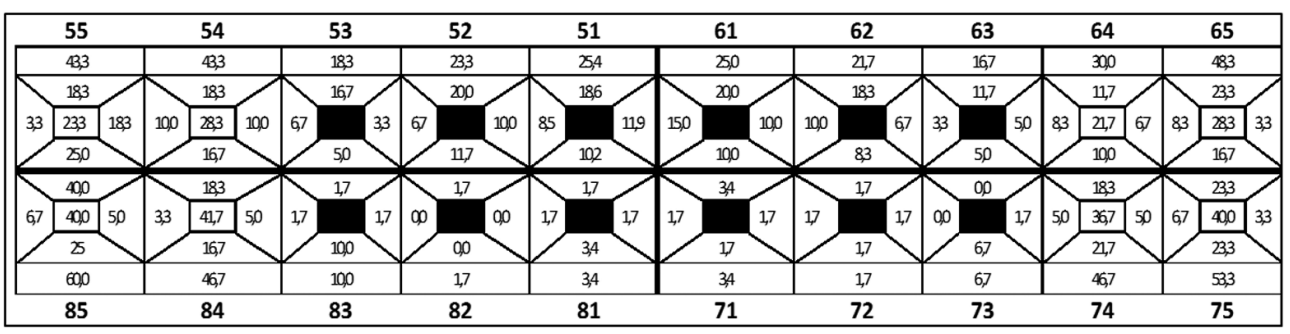

Figura 1. Experiencia de caries dental en dentición primaria, por diente y superficie (\% afectado), en niños de tres a cinco años

Fuente: elaboración propia

La experiencia de caries dental por superficies en dientes homólogos, expresada en porcentaje, se presenta en la figura 2. Allí se evidencia una mayor frecuencia de 
lesiones oclusales en los molares primarios y en las superficies bucales de incisivos y molares.

Al analizar la experiencia de caries por tipo de diente, los segundos molares inferiores fueron los más afectados, con un porcentaje de 44,3\%, seguidos por los primeros molares inferiores con 41,2 \%, los segundos molares superiores con 40,6\% y los primeros molares superiores con 35,3\%. Los incisivos y caninos superiores tuvieron valores entre 17,8 \% y 19,6\%. Los dientes anteriores inferiores presentaron los porcentajes más bajos de experiencia de caries dental: 1,4 \% para incisivos centrales, 2,3\% para incisivos laterales y 9,3\% para los caninos.

En la tabla 2 se presenta el patrón de caries para dientes homólogos, que Gizani et al (12) denominaron tasa de ataque de caries. Cada superficie fue comparada con las demás mediante la prueba Chi cuadrado o la prueba exacta de Fisher. Se encontraron diferencias estadísticamente significativas tanto en molares superiores como inferiores cuando la superficie oclusal se comparó con el resto de las superficies, con excepción del segundo molar inferior al compararla con la superficie lingual. En los dientes incisivos y caninos superiores e inferiores, la superficie bucal también mostró una mayor experiencia de caries al ser comparadas con las demás superficies. La excepción fue la superficie mesial de los incisivos centrales, donde las lesiones se presentan con más frecuencia en bucal que en mesial, pero las diferencias no fueron estadísticamente significativas.

a) Total

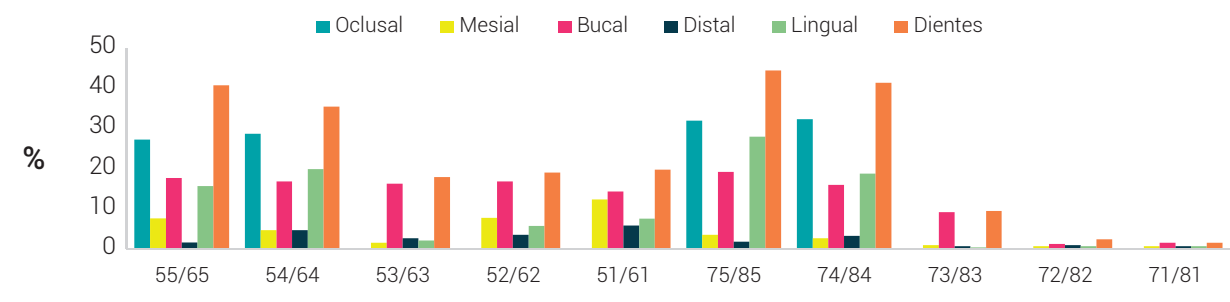

b) Tres años

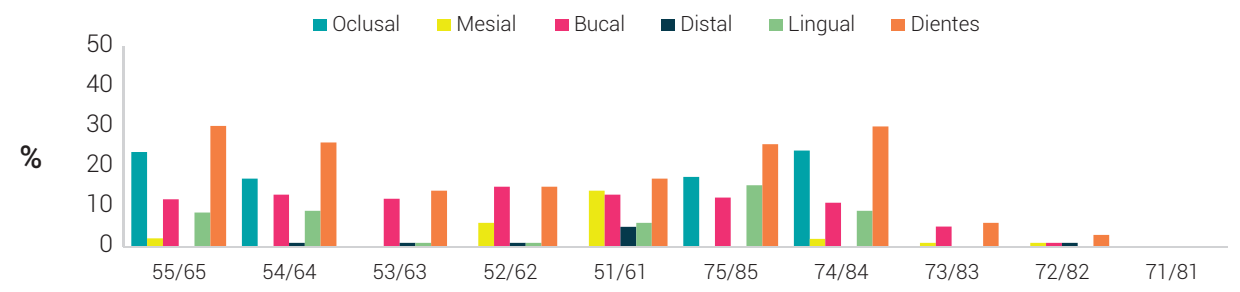


c) Cuatro años

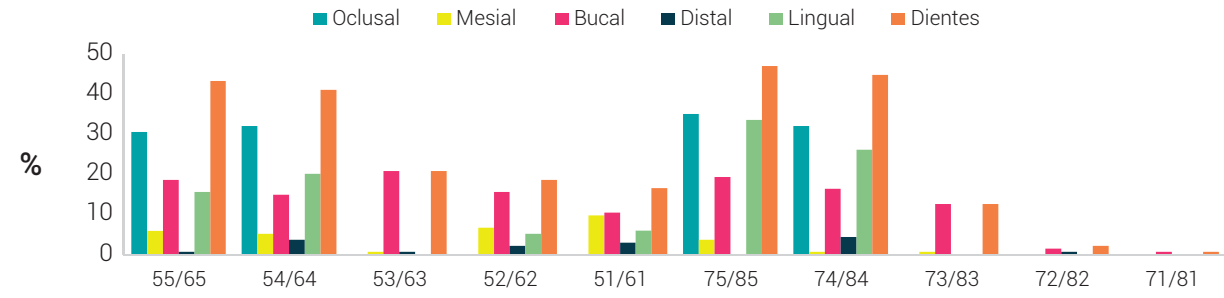

d) Cinco años

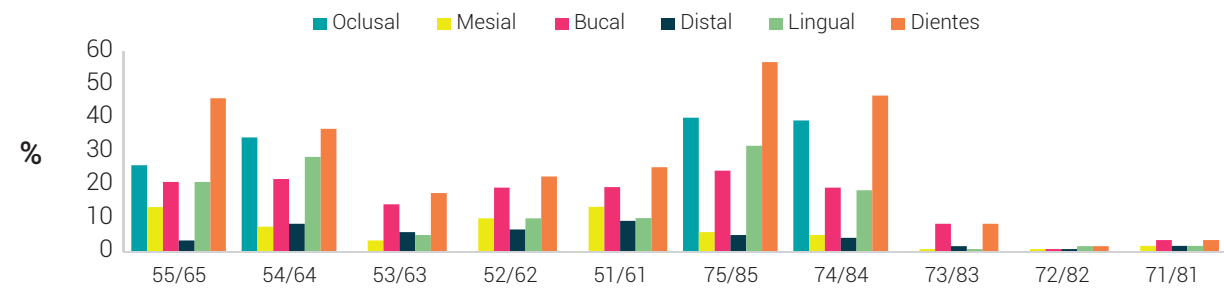

Figura 2. Distribución de experiencia de caries en dientes homólogos y por superficie afectada (\% con experiencia)

Fuente: elaboración propia

Tabla 2. Comparación de la experiencia de caries entre superficies dentales de dientes homólogos (D: distal, B: bucal, M: mesial, L: lingual, O: oclusal)

\begin{tabular}{|c|c|c|c|c|c|c|c|c|c|c|c|c|}
\hline Edad & Dientes & Patrón de caries & O-M & O-B & O-D & O-L & M-B & M-D & M-L & B-D & B-L & D-L \\
\hline \multirow{10}{*}{ TODOS } & $55 / 65$ & $O>B>L>M>D$ & $* * \star$ & $\star *$ & $\star * *$ & $\star \star \star *$ & 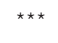 & 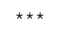 & ** & $\star * *$ & ns & $* \star *$ \\
\hline & $54 / 64$ & $O>L>B>M=D$ & $\star \star \star *$ & $\star \star \star *$ & $* * \star$ & $* *$ & $\star \star \star$ & $\mathrm{ns}$ & $\star \star * *$ & $\star \star \star *$ & ns & $\star * *$ \\
\hline & $53 / 63$ & $B>D>L>M$ & & & & & $* \star *$ & ns & ns & $\star * *$ & $* * *$ & ns \\
\hline & $52 / 62$ & $B>M>L>D$ & & & & & $* * *$ & * & ns & $* * *$ & $* * *$ & ns \\
\hline & $51 / 61$ & $B>M>L>D$ & & & & & ns & ** & * & 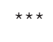 & $\star \star$ & ns \\
\hline & $75 / 85$ & $O>L>B>M>D$ & $\star \star \star$ & $\star \star * *$ & 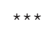 & ns & 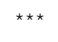 & ns & $* \star *$ & $* \star \star$ & $\star \star$ & $* * *$ \\
\hline & $74 / 84$ & $O>L>B>D>M$ & $\star \star \star *$ & 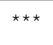 & $* \star *$ & $* \star \star$ & 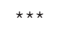 & ns & $\star \star \star *$ & 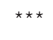 & ns & $* * *$ \\
\hline & $73 / 83$ & $B>M>D>L$ & & & & & 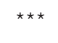 & ns & ns & 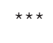 & $* \star *$ & ns \\
\hline & $72 / 82$ & $B>D>M=L$ & & & & & ns & ns & ns & ns & ns & ns \\
\hline & $71 / 81$ & $B>M=L=D$ & & & & & ns & ns & ns & ns & ns & ns \\
\hline \multirow{10}{*}{ Tres años } & $55 / 65$ & $\mathrm{O}>\mathrm{B}>\mathrm{L}>\mathrm{M}$ & $* * *$ & ns & $* * *$ & $* *$ & * & ns & ns & ** & ns & * \\
\hline & $54 / 64$ & $O>B>L>D$ & $* * *$ & ns & $\star \star *$ & ns & $* * *$ & ns & $* * *$ & $\star \star *$ & ns & * \\
\hline & $53 / 63$ & $B>L=D$ & & & & & ** & ns & ns & $\star *$ & $* *$ & ns \\
\hline & $52 / 62$ & $B>M>L=D$ & & & & & ns & ns & ns & $* * *$ & $* * *$ & ns \\
\hline & $51 / 61$ & $M>B>L>D$ & & & & & ns & ns & ns & ns & ns & ns \\
\hline & $75 / 85$ & $O>L>B$ & $* \star \star *$ & ns & $* * \star$ & ns & $\star \star *$ & $\ddagger$ & $* \star * *$ & $\star \star *$ & ns & $\star \star \star *$ \\
\hline & $74 / 84$ & $\mathrm{O}>\mathrm{B}>\mathrm{L}>\mathrm{M}$ & $* * *$ & * & $* * *$ & $* *$ & * & ns & ns & ** & ns & $* \star$ \\
\hline & $73 / 83$ & $B>M$ & & & & & ns & ns & ns & ns & ns & $\ddagger$ \\
\hline & $72 / 82$ & $B=M=D$ & & & & & ns & ns & ns & ns & ns & ns \\
\hline & $71 / 81$ & - & & & & & $\ddagger$ & $\ddagger$ & $\ddagger$ & $\ddagger$ & $\ddagger$ & $\ddagger$ \\
\hline
\end{tabular}


(viene)

\begin{tabular}{|c|c|c|c|c|c|c|c|c|c|c|c|c|}
\hline Edad & Dientes & Patrón de caries & O-M & O-B & O-D & O-L & M-B & M-D & M-L & B-D & B-L & D-L \\
\hline \multirow{10}{*}{ Cuatro años } & $55 / 65$ & $\mathrm{O}>\mathrm{B}>\mathrm{L}>\mathrm{M}>\mathrm{D}$ & $\star \star \star *$ & * & $\star \star \star *$ & $\star \star$ & $\star \star$ & * & * & 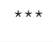 & ns & 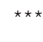 \\
\hline & $54 / 64$ & $O>L>B>M>D$ & $* \star \star$ & ** & $* * *$ & * & * & ns & $* * *$ & ** & ns & $* * *$ \\
\hline & $53 / 63$ & $B>M=D$ & & & & & $* * *$ & ns & ns & $* * *$ & $* * *$ & ns \\
\hline & $52 / 62$ & $B>M>L>D$ & & & & & * & ns & ns & $* * *$ & $\star \star *$ & ns \\
\hline & $51 / 61$ & $B>M>L>D$ & & & & & ns & * & ns & * & ns & ns \\
\hline & $75 / 85$ & $\mathrm{O}>\mathrm{L}>\mathrm{B}>\mathrm{M}$ & $\star \star *$ & ** & $\star * *$ & ns & $\star * \star$ & ns & $\star \star *$ & $* * *$ & * & $* * *$ \\
\hline & $74 / 84$ & $O>L>B>D>M$ & $* * *$ & ** & $* \star *$ & * & $* * *$ & $\mathrm{~ns}$ & $* \star *$ & ** & ns & $* * *$ \\
\hline & $73 / 83$ & $B>M$ & & & & & $\star * \star *$ & ns & ns & $* * *$ & $* \star \star *$ & $\ddagger$ \\
\hline & $72 / 82$ & $B>D$ & & & & & ns & ns & $\ddagger$ & ns & ns & ns \\
\hline & $71 / 81$ & B & & & & & ns & $\ddagger$ & $\ddagger$ & ns & ns & $\ddagger$ \\
\hline \multirow{10}{*}{ Cinco años } & $55 / 65$ & $O>B=L>M>D$ & * & ns & $* \star * *$ & ns & ns & * & ns & $* * *$ & ns & *** \\
\hline & $54 / 64$ & $O>L>B>D>M$ & 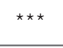 & * & $* \star *$ & ns & ** & ns & 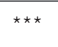 & ** & ns & *** \\
\hline & $53 / 63$ & $B>D>L>M$ & & & & & ** & ns & ns & ns & * & ns \\
\hline & $52 / 62$ & $B>L=M>D$ & & & & & ns & ns & ns & ** & ns & ns \\
\hline & $51 / 61$ & $B>M>L>D$ & & & & & ns & ns & ns & * & ns & ns \\
\hline & $75 / 85$ & $O>L>B>M>D$ & $* * *$ & * & $* \star * *$ & ns & 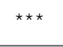 & ns & $* \star *$ & $* * *$ & ns & *** \\
\hline & $74 / 84$ & $O>B>L>M>D$ & $\star \star \star *$ & ** & 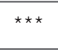 & 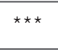 & $\star \star$ & ns & ** & $* \star \star$ & ns & ** \\
\hline & $73 / 83$ & $B>D>L=M$ & & & & & * & ns & ns & * & * & ns \\
\hline & $72 / 82$ & $L>B=M=D$ & & & & & ns & $\mathrm{ns}$ & ns & ns & ns & ns \\
\hline & $71 / 81$ & $B>L=M=D$ & & & & & ns & ns & ns & ns & ns & ns \\
\hline
\end{tabular}

${ }^{*}$ Valor $\mathrm{P}<0.05,{ }^{* *}<0.01,{ }^{* *}<0.001$ (Prueba Chi cuadrado /Prueba de Fisher)

ns: Valor $\mathrm{P}>0.05$

‡: Superficies libres de caries

Fuente: elaboración propia

\section{Discusión}

Los resultados muestran la distribución de las lesiones de caries dental en la dentición primaria en niños y niñas que asisten a un hogar infantil localizado en una zona urbana de la ciudad de Medellín. El 61 \% presenta caries de la infancia temprana y un $48 \%$ ha experimentado la forma severa de la enfermedad, de acuerdo con la definición de la Academia Americana de Odontología Pediátrica. A los tres y cinco años, la experiencia de caries fue $46 \%$ y $70 \%$ respectivamente, indicador que al ser comparado con los datos del IV Estudio Nacional de Salud Bucal de 83,03 \% y 88,83\% para esas edades, evidencia mejores indicadores en el grupo examinado.

Aunque los datos no son estrictamente comparables por las diferencias en las edades evaluadas, la cifra de $61 \%$ de experiencia de caries para todo el grupo de 
estudio es inferior al 73,87 \% reportado en el ENSAB IV para la ciudad de Medellín para las edades combinadas de uno, tres y cinco años (1). Por otro lado, a la edad de 4 años, un estudio realizado en la ciudad de Bogotá con criterios similares, mostró que un 88,5 \% de los niños tenían experiencia de caries dental (17), en comparación con $64,2 \%$ en este estudio.

Estos datos demuestran cómo, a pesar de los avances en los indicadores, la caries dental sigue presente en la mayoría de los niños y niñas que asisten de manera rutinaria a una institución de la ciudad, donde se trabaja por su desarrollo integral y se llevan a cabo acciones de cuidado. Se sabe que, en el país, solamente el 58,9\% (1) de los niños de uno, tres y cinco años han asistido a consulta odontológica. Es necesario entonces seguir trabajando en la implementación de acciones integradas con otros sectores para fortalecer prácticas de cuidado bucal y capacitación en la detección temprana de la enfermedad (3), para su control oportuno y prevención de la progresión de las lesiones.

Como en otros estudios, se encontraron lesiones de caries dental en todas las superficies examinadas, con excepción de algunas superficies de los incisivos inferiores. Los datos de experiencia de caries por diente en el grupo de estudio sugieren que las lesiones afectan con mayor frecuencia los molares primarios. Esta información es consistente con los hallazgos reportados en la misma ciudad, en un estudio que evaluó niños asistentes a centros infantiles en 2008 (13). Es difícil establecer una comparación con otros estudios, porque la información en la mayoría de ellos se presenta por dientes homólogos, a excepción de Vanobergen (11), pero en éste las prevalencias son muy bajas y se trata de un país desarrollado.

Las mayores diferencias se hacen evidentes cuando se analiza la distribución de la experiencia de caries en dientes homólogos y por superficies afectadas. La distribución no es uniforme por tipo de diente, y las diferencias entre superficies no corresponden a un patrón aleatorio. Para el grupo y en cada una de las edades, los pares homólogos de dientes más afectados fueron los molares, hallazgos que también coinciden con Sowole et al. (18), Gizzani et al. (12), y Cortés et al. (17) sobre una mayor presencia de la enfermedad en dientes posteriores.

Si bien no hay uniformidad en el uso del término patrón de caries en dentición primaria, y varios estudios asignan diferentes categorías a la agrupación de lesiones y otros sugieren que existen combinaciones de patrones (19). El término está implícito en la definición de entidades como la caries severa de la Infancia temprana que hace la AAPD, y el hecho de identificar la distribución de las lesiones en una población determinada, y las superficies más afectadas, permite enfocar de manera más eficiente los recursos para la detección y el tratamiento oportuno de las lesiones. 
En el presente estudio se hicieron evidentes las diferencias entre la superficie oclusal de los molares y la superficie bucal de dientes anteriores superiores y caninos. Esta información permite afirmar que estrategias como levantar el labio para hacer detección temprana de lesiones de caries dental (20) tienen sentido porque permiten identificar la aparición de la enfermedad y hacer una búsqueda de factores relacionados con su presencia y avance desde edades muy tempranas, pero deben ser complementadas con otras que contribuyan a identificar lesiones oclusales o mantener sanas estas superficies, como el cepillado transversal $(7,21)$.

\section{Conclusiones}

Los resultados del estudio muestran que las superficies dentales más afectadas corresponden a las oclusales de los molares, y las menos afectadas son las de los dientes anteriores inferiores. Hallazgos que presentan coincidencias con lo reportado en tres estudios en el país.

Es necesario avanzar en las estrategias educativas dirigidas a la comunidad y los equipos de salud. Algunas de ellas pueden quedarse cortas en la detección de lesiones oclusales que, según la evidencia, pudieran controlarse con métodos simples.

El presente estudio arroja datos relacionados con la distribución de las lesiones, es necesario analizar si la severidad o grado de actividad o no de las mismas en función del patrón implican modificar prioridades, para ello se requieren futuros análisis que permitan identificar las necesidades de tratamiento y las posibles estrategias para que las medidas implementadas puedan modificar el curso de la enfermedad.

\section{Agradecimientos}

Las autoras agradecen al Comité Privado de Asistencia a la Niñez -PAN por el apoyo en la ejecución del estudio.

\section{Referencias}

1. Colombia. Ministerio de Salud y Protección Social. IV Estudio Nacional de Salud BucalENSAB IV. Bogotá: 2013-2014. Recuperado en: https:/www.minsalud.gov.co/sites/rid/Lists/ BibliotecaDigital/RIDE/VS/PP/ENSAB-IV-Situacion-Bucal-Actual.pdf. Acceso Ene 2020 
2. Pitts N, Baez R, Diaz-Guallory C, Donly KJ, Feldens CA, McGrath C, et al. Early Childhood Caries: IAPD Bangkok Declaration. Int J Paediatr Dent 2019; 29: 384-6. doi: 10.1111/ipd.12490

3. World Health Organization. Ending childhood dental caries: WHO implementation manual. 2019. Recuperado en: https://apps.who.int/iris/handle/10665/330643.

4. American Academy of Pediatric Dentistry. Policy on Early Childhood Caries (ECC): Classifications, Consequences and Preventive Strategies. The Reference Manual of Pediatric Dentistry. 2019-2020. p. 74-75.

5. Nyvad B, Fejerskov O. The caries control concept. In: Fejerskov O, Nyvad B, Kidd E. Dental caries. The disease and its clinical management, (eds); $3^{\text {rd }}$ ed. Oxford: Wiley Blackwell; 2015. p. $235-243$

6. Machiulskiene V, Campus G, Carvalho JC, Dige I, Ekstrand KR, Jablonski-Momeni A, et al. Terminology of Dental Caries and Dental Caries Management: Consensus Report of a Workshop Organized by ORCA and Cariology Research Group of IADR. Caries Res. 2020; 54 : 7-14. doi: 10.1159/000503309

7. Kidd E, Fejerskov O. Essentials of dental caries. $4^{\text {th }}$ ed, Oxford University Press 2016. 196p

8. Bruzda-Zwiech A, Filipińska R, Borowska-Strugińska B, Żądzińska E, Wochna-Sobańska M. Caries experience and distribution by tooth surfaces in primary molars in the pre-school Child Population of Lodz, Poland. Oral Health Prev Dent 2015; 13: 557-66. doi: 10.3290/j.ohpd. a34371

9. Elfrink ME, Veerkamp JS, Kalsbeek H. Caries pattern in primary molars in Dutch 5-year-old children. Eur Arch Paediatr Dent. 2006;7: 236-40. doi:10.1007/BF03262558

10. Ferro R, Besostri A, Olivieri A. Caries prevalence and tooth surface distribution in a group of 5-year-old Italian children Eur Arch Paediatr Dent 2009; 10(1): 33-7. doi: 10.1007/BF03262665

11. Vanobbergen J, Lesaffre E, García-Zattera MJ, Jara A, Martens L, Declerck D. Caries Patterns in Primary Dentition in 3-5- and 7-Year-Old Children: Spatial Correlation and Preventive Consequences. Caries Res. 2007; 41(1): 16-25. doi: 10.1159/00009610

12. Gizani S, Vinckier F, Declerck D. Caries pattern and oral health habits in 2- to 6-year-old children exhibiting differing levels of caries. Clin Oral Investig. 1999; 3: 35-40. doi:10.1007/ s007840050076 
13. Escobar-Paucar GM, Ramírez-Puerta BS, Álvarez-Sánchez LG. Caries patterns in primary dentition in 3- to 5-year-old children. Medellín, Colombia. Rev Fac Odontol Univ Antioq. 2019; 31(1-2): 46-55. doi: 10.17533/udea.rfo.v31n1-2a4

14. Pitts N, Ismail Al, Martignon S, Ekstrand K, Douglas G, Longbottom C. ICCMS ${ }^{\text {TM }}$ Guide for Practitioners and Educators. International Caries Classification and Management System (ICCMSTM). Implementation Workshop held June 2013. King's College London, 2014. Recuperado en: https://www.iccms-web.com/uploads/asset/5928471279874094808086.pdf

15. Pitts NB. How the detection, assessment, diagnosis and monitoring of caries integrate with personalized caries management. In: Pitts NB (ed) Detection, assessment, diagnosis and monitoring of caries. Monographs in oral science. 21. Karger Switzerland; 2009, p. 1-14.

16. World Health Organization. Oral health surveys: Basic methods - $5^{\text {th }}$ ed. Printed in France: Présence Graphique-Monts; 2013. 125 p.

17. Cortes A, Ekstrand KR, Gamboa LF, González L, Martignon S. Caries status in young Colombian children expressed by the ICCMS ${ }^{\mathrm{TM}}$ visual/radiographic combined caries staging system. Acta Odontol Scand.2017; 75(1): 12-20. doi: 10.1080/00016357.2016

18. Sowole A, Sote E, Folayan M. Dental caries pattern and predisposing oral hygiene related factors in Nigerian preschool children. Eur Arch Paediatr Dent. 2007; 8(4): 206-10. Doi: 10.1007/ BF03262598

19. Psoter WJ, Morse DE, Pendrys DG, Zhang H, Mayne ST. Historical evolution of primary dentition caries pattern definitions. Pediatric Dentistry. 2004; 26(6): 508-11

20. Loyer-Chu L. California Childcare Health Program, Berkeley, 2007. Recuperado en: https:// cchp.ucsf.edu/sites/g/files/tkssra181/f/Oral_Health_SP_0608.pdf

21. Frazão, P. Effectiveness of the bucco-lingual technique within a school-based supervised toothbrushing program on preventing caries: a randomized controlled trial. BMC Oral Health; 2011; 11(1):11. doi: 10.1186/1472-6831-11-11 